\title{
Clinicopathological correlation in exudative age related macular degeneration: histological differentiation between classic and occult choroidal neovascularisation
}

\author{
B A Lafaut, K U Bartz-Schmidt, C Vanden Broecke, S Aisenbrey, J J De Laey, K Heimann
}

\begin{abstract}
Aims-To analyse the histopathology of classic and occult choroidal neovascular membrane surgical specimens in age related macular degeneration.

Methods-35 membranes, from a consecutive series of surgically removed choroidal neovascular membranes in age related macular degeneration, were classified as classic or occult following the guidelines of the Macular Photocoagulation Study. Membranes with classic as well as occult components were considered as mixed membranes. The membranes were serially sectioned and stained with haematoxylin and eosin, Masson trichrome, periodic acid-Schiff, and phosphotungstic acid haematoxylin stain. The correlation has been made in a masked fashion.

Results-31 membranes (19 classic, 10 occult, and two mixed membranes) could be analysed histologically. 18 classic choroidal neovascular membranes had a major subretinal fibrovascular component and 10 of these had an additional, minor fibrovascular component under the retinal pigment epithelium. The 10 occult membranes contained a fibrovascular component under the retinal pigment epithelium and the two mixed membranes contained fibrovascular tissue on both sides of the retinal pigment epithelium. Fibrin and remains of outer segments tended to occur at the lateral edges of classic membranes and to cover the inner surface of occult membranes.

Conclusion-Classic choroidal neovascularisation in age related macular degeneration is predominantly composed of subretinal fibrovascular tissue while occult choroidal neovascularisation is composed of fibrovascular tissue at the choroidal side of the retinal pigment epithelium.

(Br F Ophthalmol 2000;84:239-243)
\end{abstract}

Subfoveal choroidal neovascularisation is an important cause of visual loss in age related macular degeneration. Choroidal neovasculari- sation in age related macular degeneration, whether it appears classic or occult on fluorescein angiography, is generally considered to grow under the retinal pigment epithelium (RPE). ${ }^{1-11}$ Many authors have described the histological appearance of surgically removed choroidal neovascular membranes, ${ }^{12-19}$ but the pathological characteristics of surgically removed occult neovascularisation have not been reported yet.

Recently, neovascular membrane surgical specimens have become available as new interest has developed in the surgical removal of choroidal neovascularisation in age related macular degeneration. Foveal translocation or displacement of foveal neuroretina towards an area with healthier RPE, may offer a better functional outcome than removal of the neovascular membrane alone where the foveal neuroretina lies on a defect in the RPE. Whether eyes with classic and occult choroidal neovascularisation might benefit from this procedure is currently being investigated.

We have analysed the histological appearance of a consecutive series of neovascular membrane surgical specimens in age related macular degeneration. We report architectural differences between classic and occult choroidal neovascularisation that may lead to a better understanding of the disorder.

\section{Materials and methods}

STUDY POPULATION

The patients were at least 60 years old. They had experienced a recent drop in visual acuity (less than 3 months) due to exudative age related macular degeneration. All patients had fundus photography and fluorescein angiography not more than 14 days before surgery. Eyes with diseases predisposing to choroidal neovascularisation other than age related macular degeneration such as high myopia, multifocal choroiditis, and angioid streaks were excluded. Eyes with a serous or serohaemorrhagic pigment epithelial detachment and eyes with subretinal fibrosis or early disciform scarring were excluded. The membrane was also excluded from the analysis when the angiographic characterisation was 

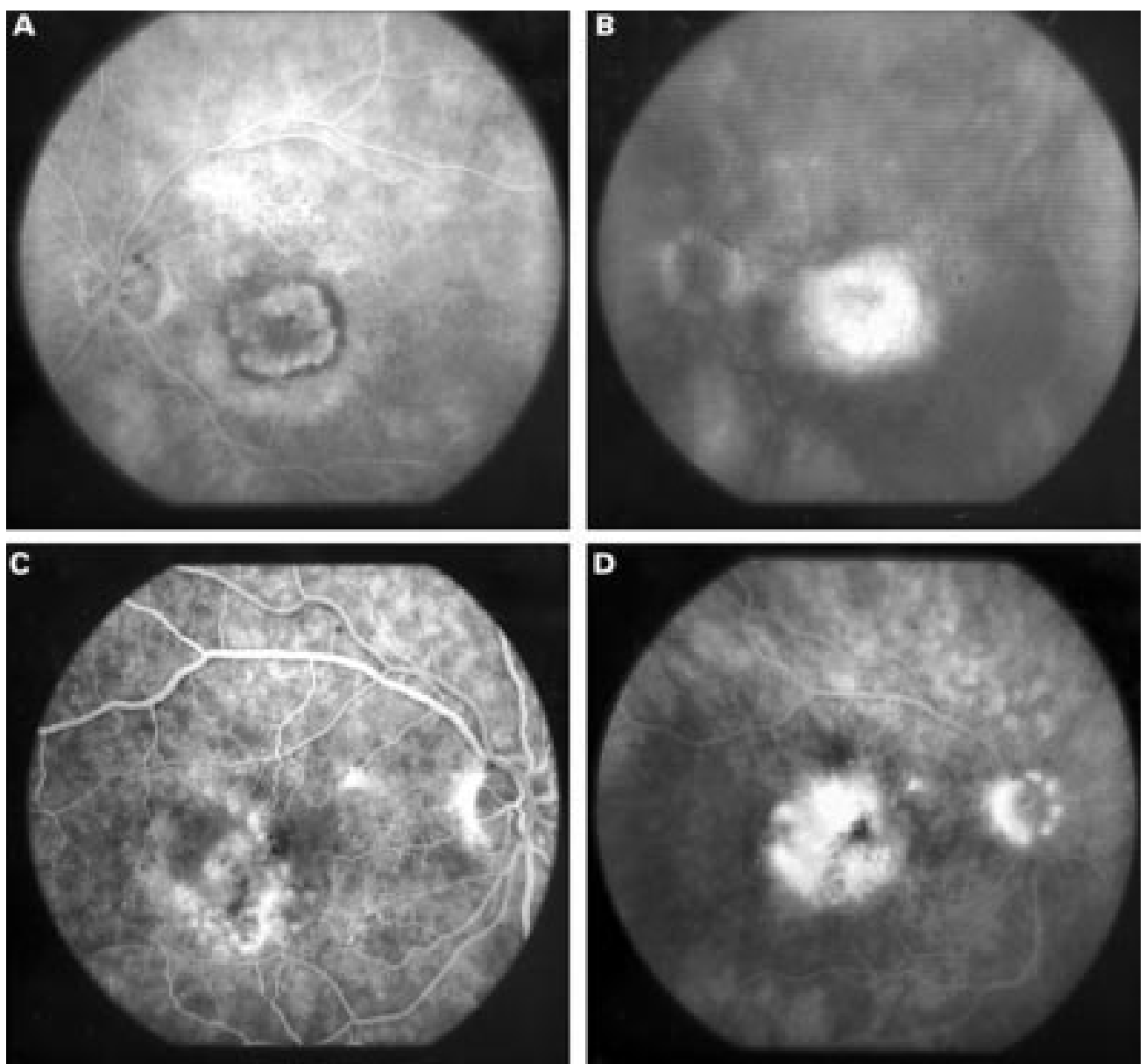

Figure 1 (A) Early phase and (B) late phase fluorescein angiogram of a classic choroidal neovascular membrane in age related macular degeneration from a 83 year old patient. (C) Early phase and (D) late phase fluorescein angiogram of an occult choroidal neovascular membrane in age related macular degeneration from a 75 year old patient.

not straightforward because of subretinal haemorrhage or cloudy media. Thirty five membranes were selected from a consecutive series of surgically removed choroidal neovascular membranes following the above mentioned criteria.

\section{ANGIOGRAPHIC INTERPRETATION}

Characterisation of the choroidal neovascularisation was performed following the guidelines of Bressler et al ${ }^{20}$ and of the Macular Photocoagulation Study. ${ }^{21}$ Choroidal neovascularisation was considered well defined or classic when a lacy net of new vessels was identified at an early stage of the fluorescein angiogram with progressive leakage later in the angiogram. Poorly defined or occult choroidal neovascularisation was characterised either by a stippled area of hyperfluorescence noted within 1-2 minutes after fluorescein injection with leakage within this area in the late phase ("fibrovascular pigment epithelial detach-

Table 1 Summary of the histopathological findings according to angiographic membrane characteristics

\begin{tabular}{lllll}
\hline & Classic & Mixed & Occult & All \\
\hline Orientation failed & $1 / 19$ & $0 / 2$ & $0 / 10$ & $1 / 31$ \\
Basal laminar deposits & $16 / 19$ & $2 / 2$ & $10 / 10$ & $28 / 31$ \\
Subretinal fibrovascular membrane & $18 / 18$ & $2 / 2$ & $2 / 10$ & $22 / 30$ \\
Sub-RPE fibrovascular membrane & $10 / 18$ & $2 / 2$ & $10 / 10$ & $22 / 30$ \\
Choroidal stroma & $3 / 18$ & $0 / 2$ & $2 / 10$ & $5 / 30$ \\
\hline
\end{tabular}

ment") or by areas of leakage at the level of the $\mathrm{RPE}$ in the late phase without well demarcated areas of hyperfluorescence discernible in the early phase ("late leakage of undetermined source"). Areas of occult choroidal neovascularisation contiguous to areas of well defined choroidal neovascularisation were defined as mixed choroidal neovascularisation and were analysed separately from occult choroidal neovascularisation.

HISTOLOGICAL ANALYSIS

The specimens were fixed in $10 \%$ neutrally buffered formalin and embedded in paraffin for light microscopy. The membranes were serially sectioned and stained in a stepped fashion with haematoxylin and eosin, Masson trichrome (MTC), and periodic acid-Schiff (PAS). Multiple sections of each membrane were stained with phosphotungstic acid haematoxylin histochemical stain for fibrin (PTAH). The primary goal of the histological analysis was to orient the membrane as being subretinal, sub-RPE, or a combination of both using two clues: (1) basal laminar deposits (BLD), a linear streak of variably granular eosinophilic material, positive on PAS stain and metachromatic blue-purple on MTC stain, reveal the choroidal side of the RPE and (2) amorphic tissue with striations perpendicular to the plane of the membrane, representing 


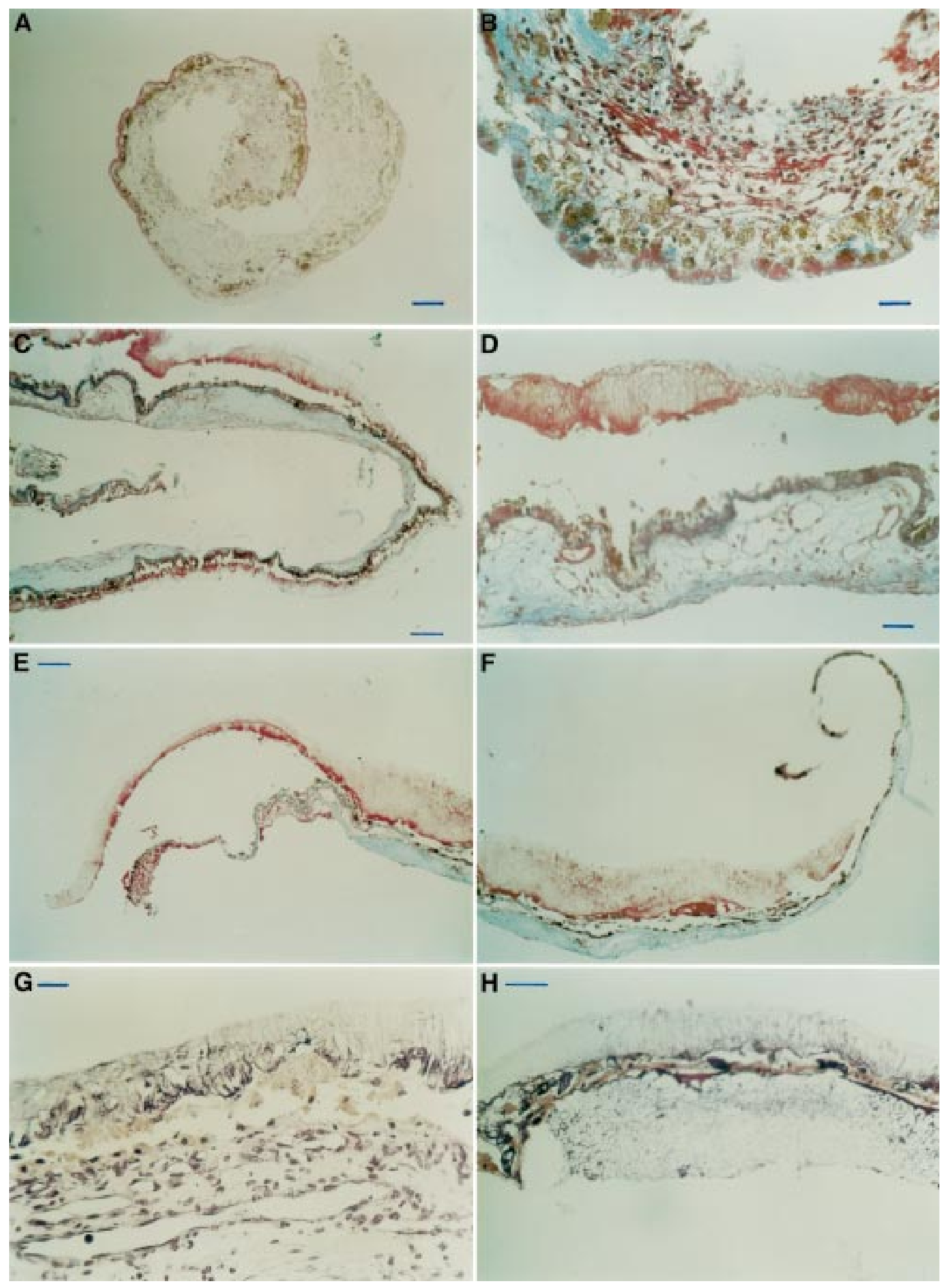

Figure 2 (A) PAS stain of a classic membrane, corresponding to Figure $1 A$ and B, indicating a major subretinal and a minor sub-RPE fibrovascular component. Both these components are separated by diffuse drusen that stained as a PAS positive line. Bar $=100 \mu \mathrm{m}$. (B) MTC stain, detail of (A). Bar $=25 \mu \mathrm{m} .(C) M T C$ stain of an occult choroidal neovascular membrane, corresponding to Figure $1 C$ and $D$, shows a sub-RPE fibrovascular membrane and remains of outer segments covering the inner surface of the membrane. Bar = $100 \mu \mathrm{m}$. (D) MTC stain, detail of (C). Bar = 25 $\mu \mathrm{m}$. (E, F) MTC stain of another occult choroidal neovascular membrane; fibrovascular tissue is seen at the choroidal side of the retinal pigment epithelium and remains of outer segment material cover the inner surface of the membrane. Bar $=100 \mu \mathrm{m} .(G)$ PTAH stain of an occult choroidal neovascular membrane. Fibrin is seen interspersed with remains of outer segments. Bar $=25 \mu \mathrm{m}$. (H) PTAH stain of an occult choroidal neovascular membrane. A serofibrinous pigment epithelial detachment is seen. Bar $=50 \mu \mathrm{m}$. 
degenerated outer segment material, indicate the neuroretinal side of the specimen. A second goal was to assess qualitatively the distribution of fibrin deposition in these specimens. Fibrin consisted of fine, eosinophilic fibrillogranular material, staining red on MTC stain. Its presence was confirmed by the PTAH stain for fibrin.

The angiographic and histological classification has been made in a masked fashion.

\section{Results}

Thirty five membranes were included in this analysis: 21 membranes were classic (Fig 1A, B), two mixed, and 12 occult (Fig 1C, D). Four membranes, two classic and two occult, were lost for histological analysis because of poor handling.

The results are summarised in Table 1 . Eighteen of the 19 remaining classic membranes could be adequately oriented with the BLD as a major clue in 16 and because of the presence of degenerated outer segment material in the absence of BLD in two others. One membrane could not be oriented. These 18 classic choroidal membranes had a major subretinal fibrovascular component (Fig 2A, B). Ten of these had an additional sub-RPE component, which was much smaller than the subretinal fibrovascular component in six membranes (Fig 2A).

The two mixed choroidal neovascular membranes were composed of obvious fibrovascular tissue on both sides of the native RPE and the BLD.

The 10 remaining occult choroidal neovascular membranes could be oriented because of the presence of RPE and diffuse drusen. A thin fibrovascular membrane was found under the RPE and the BLD in all cases (Fig 2C, D, E, F). In two cases only, an additional smaller fibrovascular component was found subretinally. This component was hypocellular and rather collagenised.

A small amount of choroidal tissue (Bruch's membrane, choriocapillaris, inner choroidal stroma) was found in five specimens, three classic and two occult membranes.

Not just capillaries but also larger calibre vessels were seen in classic membranes whereas occult membranes contained predominantly or, even, only capillaries.

Fibrin was deposited at the lateral edges of the membrane in 10 of 18 classic membranes. Remains of outer segments were often associated with these areas. Remains of outer segments interspersed with fibrin covered almost the entire inner surface only in one classic choroidal neovascular membrane. Furthermore, some fibrin deposition was often found around the native and hyperplastic RPE. In occult choroidal neovascularisation, remains of outer segments interspersed with fibrin were not only observed at the edges of the membrane but also covered its inner surface entirely in six of 10 and partially in three of 10 membranes (Fig 2G). Some fibrin was also often found around the native RPE. Areas of sub-RPE fibrovascular membrane were continuous with areas of serofibrinous detachment of the RPE in some occult membranes (Fig $2 \mathrm{H})$. Vessels were not found or only rarely seen in these areas of serofibrinous detachment of the RPE.

\section{Discussion}

Many authors have reported the histological characteristics of choroidal neovascularisation in age related macular degeneration..$^{2-19}$ The choroidal neovascular membranes in age related macular degeneration are similar to those seen in myopia, presumed ocular histoplasmosis and multifocal choroiditis except for the presence of BLD. ${ }^{7516}$ Choroidal neovascularisation conceptually represents a stereotypic wound repair like granulation response regardless of the underlying disease. ${ }^{1{ }^{16}{ }^{17}}$ Most specimens studied so far were either well defined membranes ${ }^{12}{ }^{14-19}$ or early disciform lesions. ${ }^{12} 13$ Some reported the orientation of their specimens versus the native RPE plane, characterised on the specimens by BLD. ${ }^{14-16}$ The pathology of surgical specimens from eyes with occult choroidal neovascularisation has not been reported yet.

In the present study, the histological appearance of surgically removed choroidal neovascular membranes is described and correlated with their fluorescein angiographic appearance-classic, occult, or mixed. We have used light microscopy for this analysis as the major cellular components (RPE, vascular endothelium, fibrocytes, macrophages, and photoreceptors) as well as extracellular components (collagen, BLD, and fibrin) can be correctly identified by light microscopy alone. ${ }^{16}$ The BLD are seen as an extra layer between the RPE and the outer Bruch's membrane (here defined as Bruch's membrane), which is a granular, PAS positive deposition that stains metachromatically blue-purple on MTC. It can be differentiated from the Bruch's membrane itself, which is a homogeneous linear PAS positive layer. The histopathological interpretation of surgical specimens is difficult; distortion of specimens can often only be overcome by serial sectioning and incomplete removal of the membrane cannot be excluded.

We have observed histoarchitectural differences between classic and occult choroidal neovascularisation. The 18 classic choroidal neovascular membranes have a major subretinal fibrovascular component and only 10 of these have an additional minor sub-RPE component. In contrast, the 10 occult choroidal neovascular membranes have a sub-RPE fibrovascular component and in two of them an additional thin fibrous subretinal membrane is seen. The two mixed choroidal neovascular membranes were composed of both subretinal as well as sub-RPE fibrovascular components. Other histological characteristics may additionally contribute to the angiographic differences: accumulation of degenerated outer segment material interspersed with fibrin tends to be seen at the lateral edges of classic membranes, corresponding to "the rim" reported by Lopez et $a l^{19}$ while such material is more diffusely distributed over the entire surface of occult choroidal neovascular membranes. 
These findings challenge the concept that choroidal neovascularisation in age related macular degeneration grows under the RPE. ${ }^{1-11}$ Choroidal neovascularisation in age related macular degeneration probably starts as sub$\mathrm{RPE}$ fibrovascular tissue. It will probably be visible as occult choroidal neovascularisation when it continues to grow in the sub-RPE space but will appear classic or mixed when it grows through the plane of the native RPE and proliferates in the subretinal space.

Gass $^{5}{ }^{6}$ suggested that choroidal neovascularisation in age related maculopathy grows primarily under the RPE (type 1), in contrast with choroidal neovascularisation in younger patients with presumed ocular histoplasmosis syndrome or with other disorders, where it occurs in the subretinal space (type 2). By accepting this architectural difference, he could intuitively explain the striking difference in surgical results between the two groups. $\mathrm{He}$ related the angiographic appearance to a difference in blood flow rate: the blood flow is slow in occult choroidal neovascularisation. With an increase in blood flow through the network with a thinned overlying RPE, classic neovascularisation may be observed. ${ }^{6}$

Grossniklaus and Green ${ }^{15}$ were able to orient 32 surgical specimens from patients with new subfoveal choroidal neovascularisation, recurrent subfoveal choroidal neovascularisation, or large subfoveal subretinal haemorrhages due to age related macular degeneration for the submacular surgery trial. Sixteen membranes had a sub-RPE fibrovascular component and 29 contained a subretinal fibrovascular component. Sixteen membranes were entirely subretinal. They suggested that more specimens may have had a sub-RPE component owing to the excision of subretinal component only. These results ${ }^{15}$ as well as others ${ }^{16}$ already indicated that subretinal neovascularisation is a more common component of choroidal neovascularisation in age related macular degeneration than was previously thought ${ }^{1-8}$ and are concordant with our findings in well defined choroidal neovascularisation.

In conclusion, the present results offer an alternative, architectural explanation for the angiographic appearance of classic and occult choroidal neovascularisation in age related macular degeneration. Classic membranes probably grow above the native RPE and are often associated with a smaller fibrovascular component under the RPE. Occult membranes grow under the RPE and BLD.

1 Bressler NM, Bressler SB, Fine SL. Age-related macular degeneration. Surv Ophthalmol 1988;32:375-413.

2 Bressler SB, Silva JC, Bressler NM, et al. Clinicopathologic correlation of occult choroidal neovascularization in age-related macular degeneration. Arch Ophthalmol 1992; 110:827-32.

3 Chang TS, Freund KB, De La Cruz Z, et al. Clinicopathologic correlation of choroidal neovascularization demonstrated by indocyanine green angiography in a patient with retention of good vision for almost four years. Retina 1994 ; 14:114-24.

4 Gass JDM. Pathogenesis of disciform detachment of the neuroepithelium. I. General concepts and classification. Am f Ophthalmol 1967;63:573-85; 617-44.

5 Gass JDM. Biomicroscopic and histopathologic considerations regarding the feasibility of surgical excision of tions regarding the feasibility of surgical excision of
subfoveal neovascular membranes. Am f Ophthalmol 1994; 118:285-98.

6 Gass JDM. Stereoscopic atlas of macular diseases. 4 th ed. St Gass JDM. Stereoscopic atlas of
Louis: Mosby, 1997:26-32, 84 .

7 Green WR, McDonnell PJ, Yeo JH. Pathologic features of senile macular degeneration. Ophthalmology 1985;92:61527

8 Green WR, Enger C. Age-related macular degeneration histopathologic studies. The 1992 Lorenz E. Zimmerman lecture. Ophthalmology 1993;100:1519-35.

9 Sarks SH. New vessel formation beneath the retinal pigment epithelium in senile eyes. Br f Ophthalmol 1973;57:951-65.

10 Sarks JP, Sarks SH, Killingsworth MC. Morphology of early choroidal neovascularization in age-related macular degeneration: correlation with activity. Eye 1997;11:51522 .

11 Small ML, Green WR, Alpar JJ, et al. Senile macular degeneration. A clinicopathologic correlation of two cases with neovascularization beneath the retinal pigment epithelium. Arch Ophthalmol 1976;94:601-7.

12 Das A, Puklin JE, Frank RN, et al. Ultrastructural immunocytochemistry of subretinal neovascular membranes in age-related macular degeneration. Ophthalmology 1992;99: 1368-76.

13 De Juan E, Machemer R. Vitreous surgery for hemorrhagic and fibrous complications of age-related macular degeneration. Am f Ophthalmol 1988;105:25-9.

14 Grossniklaus HE, Gass JDM. Clinicopathologic correlation of surgically excised type 1 and type 2 submacular choroidal neovascular membranes. Am f Ophthalmol 1998;126: 59-69.

15 Grossniklaus HE, Green G, the Submacular Surgery Trials Research Group. Histopathologic and ultrastructural findings of surgically excised choroidal neovascularization. Arch Ophthalmol 1998;116:745-9.

16 Grossniklaus HE, Hutchinson AK, Capone A, et al. Clinicopathologic features of surgically excised choroidal neovascular membranes. Ophthalmology 1994;101:1099-111.

17 Grossniklaus HE, Martinez JA, Brown VB, et al. Immunohistochemical and histochemical properties of surgically excised subretinal neovascular membranes in age-related macular degeneration. Am f Ophthalmol 1992;114:464-72.

18 Lopez PF, Grossniklaus HE, Lambert HM, et al. Pathologic features of surgically excised subretinal neovascular membranes in age-related macular degeneration. Am $\mathcal{f}$ Ophthalmol 1991;112:647-56.

19 Lopez PF, Lambert M, Grossniklaus HE, et al. Well-defined choroidal neovascular membranes in age-related macular degeneration. Ophthalmology 1993;100:415-22.

20 Bressler NM, Bressler SB, Gragoudas ES. Clinical characteristics of choroidal neovascular membranes. Arch Ophteristics of choroidal neovasc

21 Macular Photocoagulation Study Group. Subfoveal neovascular lesions in age-related macular degeneration. Guidelines for evaluation and treatment in the Macular Photocoagulation Study. Arch Ophthalmol 1991;109:1242-57. 